\title{
EL DERECHO COMUNITARIO ANDINO Y SU CONTROL JURISDICCIONAL"
}

\author{
Aída Fernández de los Campos \\ Emma Elvira Ortiz Arciniegas \\ Recibido: Julio 2 de 2015 \\ Aprobado: Noviembre 25 de 2015
}

\begin{abstract}
RESUMEN
Este artículo abarca el Derecho Comunitario Andino y sus principios, el Tribunal de Justicia de la Comunidad Andina y sus funciones jurisdiccionales. Constituye un avance del proyecto de investigación "El Derecho Comunitario Andino: Desarrollos jurisprudenciales del Tribunal de Justicia de la Comunidad Andina”, el cual se centra en torno a la pregunta acerca de cuáles son los criterios jurisprudenciales en el período 2008-2012 de dicho órgano, respecto a la interpretación prejudicial y a la acción de incumplimiento, dos de las competencias atribuidas por los Estados Miembros en los tratados constitutivos de la Organización. Este trabajo contribuye a la generación de nuevo

\footnotetext{
* Avance del proyecto de investigación intitulado "El Derecho Comunitario Andino: desarrollos jurisprudenciales del Tribunal de Justicia de la Comunidad Andina", inscrito al Centro de Estudios Latinoamericanos, Grupo de Investigación de "Hermenéutica Jurídica", línea de investigación análisis jurídico, adscrito al Centro de Investigaciones Sociojurídicas “Laureano Gómez Serrano" de la Facultad de Derecho de la Universidad Autónoma de Bucaramanga, UNAB.

** Doctora en Derechos Humanos y Desarrollo de la Universidad Pablo de Olavide de Sevilla, España. Magistra en Derecho de Familia de la Universidad Autónoma de Bucaramanga, UNAB, Doctora en Derecho y Ciencias Sociales de la Universidad de la República, Uruguay. Docente titular, coordinadora de la Línea de Derecho Público y del Centro de Estudios Latinoamericanos de la Facultad de Derecho de la UNAB. Correo electrónico: demafc@unab.edu.co

*** Diploma de Estudios Avanzados del Programa de Doctorado en Derecho de la Universidad de Alcalá, España. Abogada de la Facultad de Derecho de la Universidad Autónoma de Bucaramanga, UNAB. Especialista en Derecho Público y en Educación con nuevas tecnologías de la UNAB. Docente de pregrado y posgrado de la UNAB y coordinadora de la modalidad virtual de la Facultad de Derecho de la UNAB. Correo electrónico:eortiz@unab.edu.co
} 
conocimiento en relación con el ordenamiento jurídico andino, en aras de la correcta aplicación de las normas comunitarias y la existencia de seguridad jurídica en la subregión andina.

Palabras claves: Comunidad Andina, Derecho Comunitario Andino, Tribunal de Justicia de la Comunidad Andina, Interpretación Prejudicial Acción de Incumplimiento.

\title{
THE ANDEAN COMMUNITY LAW AND THE JURISDICTIONAL CONTROL OF ITS APPLICATION
}

\begin{abstract}
This article focuses on the Andean Community Law and its principles, the Court of Justice of the Andean Community and its rulings. It constitutes a preview of the research project, "The Andean Community Law: Case law from the Court of Justice of the Andean Community", and seeks to identify the case law criteria of this body within the period 2008-2012, regarding preliminary rulings and infringement proceedings, two of the functions attributed to it by the Member States in the organization's constituent instruments. This project contributes to generate new knowledge regarding the Andean Community Law in order to ensure that it is interpreted and applied the same in every Andean Community country.
\end{abstract}

Key words: Andean Community, Andean Community Law, Court of Justice of the Andean Community, Preliminary Rulings Infringement Proceedings.

\section{O DIREITO COMUNITÁRIO ANDINO E SEU CONTROLE JURISDICIONAL}

\section{RESUMO}

Este artigo compreende o Direito Comunitário Andino e seus princípios, o Tribunal de Justiça da Comunidade Andina e suas funções jurisdicionais. É uma etapa do projeto de pesquisa "O Direito Comunitário Andino: Desenvolvimento jurisprudencial do Tribunal de Justiça da Comunidade Andina", que gira em torno da questão dos critérios legais deste órgão, no período de 2008-2012, a respeito da interpretação preliminar e da ação de não cumprimento, dois dos poderes conferidos pelos Estados-Membros nos Tratados da 
Organização. Este trabalho contribui para a geração de novos conhecimentos sobre o sistema jurídico andino, a fim de assegurar a correta aplicação das normas comunitárias e da existência de segurança jurídica na sub-região andina.

Palavras-chave: Comunidade Andina, Direito Comunitário Andino, Tribunal de Justiça da Comunidade Andina, Interpretação Prejudicial, Ação de Não cumprimento

\section{INTRODUCCIÓN}

La actual era de la globalización, caracterizada por una creciente gravitación de los procesos financieros, económicos, ambientales, políticos y culturales de alcance mundial en los de carácter regional, nacional y local; genera una desigual participación entre los actores que constituye un desafío para los pueblos del planeta.

Los países en vías de desarrollo, como los latinoamericanos, grupo al cual pertenece Colombia, deben adoptar una nueva visión de la realidad mediante la búsqueda de modelos alternativos que les permitan aprovechar las ventajas de la globalización. Es así como los Estados encuentran en la integración, una forma de enfrentarla en términos favorables, con la finalidad de procurar un mejoramiento persistente en el nivel de vida de sus habitantes, como en el caso de la Comunidad Andina.

La Comunidad Andina se funda en un origen provisto de rasgos comunes de los siguientes Estados: Bolivia, Perú, Colombia y Ecuador. Su vínculo de pertenencia constituye un conjunto de relaciones y situaciones reguladas por un orden jurídico común, el derecho andino, el cual presenta unas características que le otorgan identidad propia, con el objeto de surtir plenos efectos y de permitir a los sujetos del Derecho Comunitario andino, es decir a los Estados Miembros, los órganos e instituciones del Sistema Andino de Integración (SAI) y a los particulares, la realización plena de los derechos derivados de este ordenamiento, además de coadyuvar al cumplimiento de las orientaciones originadas en el mismo.

El Tribunal de Justicia de la Comunidad Andina garantiza la interpretación y aplicación uniforme de la normativa jurídica andina, y 
en caso de entrar en conflicto las relaciones intersubjetivas de intereses que dicho orden disciplina, asegura la efectividad de la tutela de los derechos.

Este artículo que se centra en la Comunidad Andina, cuyo ordenamiento jurídico está sometido al control jurisdiccional del Tribunal de Justicia de la Comunidad Andina, constituye un avance del proyecto de investigación" intitulado "El Derecho Comunitario Andino: desarrollos jurisprudenciales del Tribunal de Justicia de la Comunidad Andina", del Centro de Estudios Latinoamericanos, como parte del Grupo de Investigación de "Hermenéutica Jurídica", de la línea de investigación análisis jurídico, adscrito al Centro de Investigaciones Sociojurídicas "Laureano Gómez Serrano" de la Facultad de Derecho de la Universidad Autónoma de Bucaramanga, UNAB.

Para la elaboración de este escrito, se parte del concepto de integración, se hace referencia a la Comunidad Andina como proceso de integración latinoamericana, se describe al Derecho Comunitario Andino, sus características y principios, y el control jurisdiccional del Tribunal de Justicia de la Comunidad Andina, con especial referencia a la interpretación prejudicial y a la acción de incumplimiento.

\section{2. ¿QUÉ SE ENTIENDE POR INTEGRACIÓN?}

De acuerdo con el Diccionario de la Real Academia Española ${ }^{2}$, integrar es la acción y el efecto de constituir un todo con las partes. En esta misma línea, Sáchica (1990) explica que "dicha acción se dirige a obtener una articulación funcional de elementos, los cuales se consideran partes de un mismo ser de actores necesarios para lograr unidad de dirección con base en un propósito común, sin perder su anterior identidad".

Por lo tanto, la integración es un concepto que sugiere imagen de totalidad, de comprensión unificadora, sin perjuicio de la variedad. Nacida de una decisión política y con proyecciones de igual índole, es fruto de maduración, intento de construir el porvenir, planificándolo. Constituye la concertación deliberada entre varias unidades autónomas

\footnotetext{
1 Para la realización de esta investigación de tipo descriptivo, con técnicas de revisión documental, se emplea el método deductivo. El trabajo comprende las siguientes etapas que se cumplirán en veinticuatro meses: recopilación de sentencias del período 2008-2012 del Tribunal de Justicia de la Comunidad Andina, respecto a la interpretación prejudicial y a la acción de incumplimiento, mediante la observación directa; lectura de los documentos, sistematización, interpretación de resultados y conclusiones. De esta manera, al final de la investigación se identificarán los criterios jurisprudenciales en el lapso delimitado.

2 Sitio Web Real Academia Española. Recuperado de http://www.rae.es/rae.html
} 
de un programa de acción conjunta, para lo cual crean una organización independiente con una finalidad específica, la integración, dotándola de poderes y recursos para lograrla. Se crea un único y nuevo centro de decisión, con competencia de atribución específica en diferentes ámbitos, con fuerza subordinante sobre los Estados que transfirieron dichas atribuciones, y, por consiguiente, con jurisdicción espacial en el ámbito territorial de éstos (Sáchica, 1990).

Dentro de este propósito caben múltiples modelos de integración, según se quiera más intensa, acelerada, global o sectorial, constituyendo la Unión Europea $^{3}$ el proceso más avanzado en la actualidad. En Latinoamérica se encuentra la Comunidad Andina ${ }^{4}$, inspirada en el modelo europeo como proceso de integración subregional, en el marco de la Asociación Latinoamericana de Integración (ALADI) ${ }^{5}$.

Es menester destacar, que desde la perspectiva de los países en vías de desarrollo los latinoamericanos, entre los que se encuentra Colombia, la integración ${ }^{6}$ supone una forma de enfrentar la era de la globalización, proceso dinámico y multidimensional, que sacude actualmente al mundo donde se produce una creciente gravitación de los procesos financieros, económicos, ambientales, políticos y culturales, de alcance mundial en los de carácter regional, nacional y local, que origina una tensión entre las diferentes dimensiones con una participación desigual entre los actores ${ }^{7}$.

\section{LA COMUNIDAD ANDINA, MANIFESTACIÓN DEL PROCESO DE INTEGRACIÓN EN LATINOAMÉRICA}

Troconis Villarreal (2004) señala que comunidad deriva de communitas; communitas, de communis; communis, de munus; y munus, cuya raíz mei denota "intercambio", se asocia a onus y a officium (Paul., en Dig. 50,16,18). También recuerda que Quintiliano en sus Instituciones

3 Sitio Web Unión Europea. Recuperado de http://europa.eu/index_es.htm

4 Sitio Web ComunidadAndina. Recuperado de http://www.comunidadandina.org/

5 Organización regional, creada mediante el Tratado de Montevideo de 1980, en reemplazo de la Asociación Latinoamericana de Libre Comercio (ALALC), Actualmente sus miembros son: Argentina, Bolivia, Brasil, Chile, Colombia, Cuba, Ecuador, México, Panamá, Paraguay, Perú, Uruguay y Venezuela. Sitio Web ALADI. Recuperado de http://www.aladi.org/

6Los Estados encuentran en la integración, una forma de enfrentar la globalización en términos favorables, pues constituye un proceso multidimensional, aunque inicialmente económico, comprende varios ámbitos, tendentes a lograr la comunidad y la unión de los Estados, con la finalidad de procurar un mejoramiento persistente en el nivel de vida de sus habitantes, como en el caso de la Comunidad Andina.

7Ver el informe anual de la CEPAL, Panorama Social de América Latina 2004. Recuperado de http:// www.eclac.cl/cgi-bin/getProd.asp?xml=/publicaciones/xml/6/20386/P20386.xml\&xsl=/dds/tp1/p9f.xs1 
$(7,3,24)$ enseña que lo común empieza donde lo propio termina. La carga de la communitas es una carga común, una tarea común, la cual en el caso de la Comunidad Andina, es la del desarrollo económico y social.

En este mismo sentido, respecto al fenómeno comunitario, el Tribunal de Justicia de la Comunidad Andina expresa que "entre nosotros nos muestra a los Estados de la región y de la subregión Andina, por conducto de sus plenipotenciarios y directamente por sus jefes de Estado, como voceros de los países, que en uso precisamente de sus poderes soberanos individuales, manifiestan su disposición de asociarse con otros Estados en un esfuerzo común a fin de hacer frente a los retos del mundo contemporáneo. Deciden de consuno acometer las tareas del desarrollo económico y el progreso social en materias que rebasan el espacio interno para constituirse en objeto de la organización común"”.

Los habitantes de los Estados Miembros, tienen entre otros, un doble vínculo de pertenencia: el vínculo primero, con la Nación, y el segundo, con la Comunidad, con la Comunidad Andina ${ }^{10}$, que es $a b$ inis una Comunidad de Naciones. Se fundan en un origen provisto de rasgos comunes, comparten communis periculi y, se apoyan en una communis fides, aspiran a una comunidad de destino.

\subsection{El Derecho Comunitario Andino y sus principios}

El Derecho Comunitario Andino, como todo ordenamiento jurídico común, es un sistema normativo estructurado e institucionalizado que contiene poderes y competencias propias, atribuidas por los Estados Miembros de la Comunidad Andina en el ejercicio de su soberanía y plasmados en sus tratados constitutivos y protocolos modificatorios.

Dicho ordenamiento comprende, de conformidad con el Tratado de Creación del Tribunal de Justicia de la Comunidad Andina, art. 1: el Acuerdo de Cartagena, sus protocolos e instrumentos adicionales; el propio Tratado de Creación del Tribunal de Justicia y sus protocolos modificatorios; las decisiones del Consejo Andino de Ministros de Relaciones Exteriores y la Comisión de la Comunidad Andina; las

\footnotetext{
9 Tribunal de Justicia de la Comunidad Andina. Sentencia del Proceso 1 - IP- 96, publicada en la G.O.A.C. No. 257 del 14 de abril de 1997.

10 La Comunidad Andina engloba un espacio de $3.798 .000 \mathrm{~km} 2$, su población es de 100 millones de habitantes, concentra el $20 \%$ de la biodiversidad de la totalidad del planeta, el $10 \%$ del agua dulce del mundo y el $35 \%$ de los bosques de América Latina y el Caribe, según datos del Sitio Web de la CAN. Recuperado de http://www.comunidadandina.org/Quienes.aspx
} 
resoluciones de la Secretaría General y los convenios de complementación industrial y otros que adopten los Estados miembros entre síen el marco del esquema subregional.

Se trata, al igual que los ordenamientos jurídicos de los países miembros, de una estructura supraordenada, cuya jerarquía superior está compuesta por normas jurídicas constitutivas, originarias o primarias respecto de las normas derivadas, las cuales están subordinadas al derecho originario.

Tal como se establece en el Estatuto del Tribunal de Justicia Andino ${ }^{11}$, las normas jurídicas constitutivas, originarias o primarias son: el Acuerdo de Cartagena, sus protocolos modificatorios e instrumentos adicionales, y el Tratado de Creación del Tribunal de Justicia de la Comunidad Andina y sus Protocolos modificatorios e instrumentos adicionales.

Las derivadas están conformadas por las decisiones del Consejo de Ministros de Relaciones Exteriores y de la Comisión de la Comunidad Andina, las resoluciones de la Secretaría General y los Convenios de Complementación Industrial.

Es de hacer notar que las fuentes derivadas, a diferencia de las originarias que provienen de los acuerdos constitutivos y que por tanto son convencionales, son producto de un proceso legislativo por parte de los respectivos órganos supranacionales de la Comunidad Andina ${ }^{12}$, a los cuales se les ha transferido competencias para tal efecto.

Además de las fuentes primarias y derivadas, el Derecho Comunitario Andino tiene fuentes no normativizadas, tales como las que emergen de los principios generales del derecho y de la jurisprudencia comunitaria.

\subsection{Los principios del Derecho Comunitario Andino}

El ordenamiento jurídico andino se caracteriza por la supranacionalidad, la autonomía, la preservación y el complemento indispensable (Tangarife Torres, 2005, p.282).

La supranacionalidad del ordenamiento jurídico andino comporta los siguientes elementos: a) La creación de un organismo sujeto de derecho internacional; b) el traslado de competencias soberanas de los Estados

11 Consejo Andino de Ministros de Relaciones Exteriores. Decisión 500 del 22 de junio de 2001. Recuperado de http://www.comunidadandina.org/Normativa.aspx\#

12 El Consejo de Ministros de Relaciones Exteriores, la Comisión y la Secretaría General de la Comunidad Andina. 
Miembros a órganos creados dentro del organismo internacional, por decisión autónoma y soberana de cada uno de ellos; c) el ejercicio de competencias por parte de los órganos creados dentro del organismo internacional, con efectos vinculantes para los sujetos del Derecho Comunitario; la transformación del concepto tradicional del Estado nación por uno que le permite ejercer decisiones soberanas que son fuentes de derechos y obligaciones en el marco de un organismo internacional; e) la personalidad limitada a los objetivos propuestos dentro del proceso de integración del que goza el organismo internacional y f) las competencias limitadas y otorgadas expresamente a los órganos e instituciones del organismo internacional que encarna los propósitos e instrumentos de dicha estructura (Uribe Restrepo, 1990, pp.49-50).

Respecto de la autonomía, el ordenamiento jurídico andino otorga a los órganos e instituciones del Sistema Andino de Integración la independencia necesaria frente a las autoridades de los Países Miembros para cumplir sus funciones, con fundamento en reglas propias que no están condicionadas por lo que pueda señalar la normativa interna de cada uno de ellos.

La preservación se sustenta en los principios y obligaciones contenidos en el Tratado de Creación del Tribunal de Justicia de la Comunidad Andina, en el artículo $4^{\circ}$, así como en las competencias que se le atribuyen a este órgano jurisdiccional de las cuales derivan el conocimiento y decisión de los recursos y acciones previstos en el referido Tratado. Esta cualidad implica que el ordenamiento jurídico andino es de carácter vinculante e intangible ${ }^{13}$.

Las normas del ordenamiento jurídico andino son obligatorias, ya que provienen de la decisión soberana de los Estados Miembros de dar origen a la Comunidad Andina y a sus órganos e instituciones, transfiriéndose competencias propias de los órganos nacionales a órganos comunitarios supranacionales y por ende, adquiriendo derechos y contrayendo obligaciones de hacer y de no hacer frente a los demás socios comunitarios.

13 En el Proceso 16-AI-99, publicado en la G.O.A.C. No. 581 de 16 de julio de 2000, el Tribunal de Justicia señala que mediante este artículo, los países miembros han adquirido "doble obligación: una de carácter positivo de 'hacer' y otra de orden negativo de 'no hacer'. Por la primera los países miembros deben adoptar toda clase de medidas que garanticen el cumplimiento de la normativa andina, es decir, de las obligaciones y compromisos adquiridos en virtud del derecho originario y de las que corresponda por mandato de las normas secundarias o derivadas. Por otra parte en virtud de la segunda obligación el país miembro debe abstenerse de toda medida así sea legislativa, judicial, ejecutiva o administrativa de orden central o descentralizado geográficamente o por servicios, llámese leyes, reglas, procedimientos, requisitos, decisiones, decretos, resoluciones, acuerdos, dictámenes, sentencias o providencias que puedan obstaculizar la aplicación del ordenamiento jurídico andino". 
La intangibilidad hace referencia a que los gobiernos de los Estados Miembros tienen vedada la posibilidad de modificar unilateralmente las normas adoptadas en el marco de la Comunidad Andina, ya que de hacerlo incurren en responsabilidad internacional para su Estado.

El complemento indispensable alude a la prohibición de los Estados Miembros para interpretar o reglamentar las normas jurídicas andinas. Solamente, y aplicando criterios restrictivos de interpretación, cabe la reglamentación de normas andinas mediante "medidas" internas, encaminadas a garantizar la correcta aplicación del ordenamiento jurídico andino, y que no sea contrario a este último ${ }^{14}$.

\subsection{El Tribunal de Justicia de la Comunidad Andina}

A medida que el proceso de integración fue evolucionando hacia su institucionalización como organismo supranacional, se tornó imperativo contar con un órgano jurisdiccional, con el fin de armonizar y uniformizar la declaración e interpretación de la normativa jurídica comunitaria para su aplicación también uniforme en todos los Estados Miembros de la Comunidad Andina.

El Tribunal se constituyó mediante el Tratado de Creación del Tribunal del Acuerdo de Cartagena de 28 de mayo de 1979, modificado por el Protocolo de Cochabamba el 28 de mayo de 1996 bajo el actual nombre de Tribunal de Justicia de la Comunidad Andina, cuya normativa ha sido codificada por la Comisión mediante la Decisión 472 de 16 de septiembre de 1999. Su Estatuto fue aprobado mediante la Decisión 184 de fecha 19 de agosto de 1983 y, posteriormente modificado y codificado por la Decisión 500 del Consejo Andino de Ministros de Relaciones Exteriores de fecha 22 de junio de 2001. Por medio de la Decisión 633 de fecha 12 de junio de 2006, este Consejo decidió que el número de Magistrados que integren el Tribunal de Justicia de la Comunidad Andina fuese igual al número de Países Miembros de la Comunidad Andina, por lo que en la actualidad son cuatro ${ }^{15}$.

14 En Sentencia dictada en el expediente No. 10-IP-94, del 17 de marzo de 1995, publicada en la G.O.A.C. No. 177, del 20 de abril del mismo año, el Tribunal de Justicia expresa "En relación con el régimen descrito, debe reiterarse el alcance de "complemento indispensable" que, en relación con la norma comunitaria, se atribuye a la norma nacional. A propósito del "complemento indispensable", este Tribunal ha declarado que, en su aplicación, "las legislaciones internas de cada país no podrán establecer exigencias, requisitos adicionales o dictar reglamentaciones que de una u otra manera entren en conflicto con el derecho comunitario andino o restrinjan aspectos esenciales regulados por él de manera que signifiquen, por ejemplo una menor protección a los derechos consagrados por la norma comunitaria".

15 Carlos Jaime Villarroel Ferrer (Bolivia), Leonor Perdomo Perdomo (Colombia), José Vicente Troya Jaramillo (Ecuador) y Ricardo Vigil Toledo (Perú). 
Los Magistrados son designados por la Comisión de las ternas presentadas por cada uno de los Países Miembros; es parte integrante del Sistema Andino de Integración (SAI), pero al mismo tiempo independiente de los órganos que lo conforman, de los Países Miembros y de las instituciones. Por consiguiente, es parte del orden jurídico comunitario, constituyéndose en la médula de un Poder Judicial de la Comunidad Andina de carácter supranacional ${ }^{16}$; su sede permanente se encuentra en la ciudad de Quito, Ecuador.

\subsection{Funciones jurisdiccionales del Tribunal de Justicia de la Comunidad Andina}

De acuerdo con lo establecido en el Tratado de Creación ${ }^{17}$ y en su Estatuto $^{18}$, el Tribunal de Justicia carece de competencias generales, por lo que solo puede ejercer las que le han sido expresamente atribuidas en forma de acciones y recursos. Por consiguiente, le corresponde interpretar y declarar el Derecho Comunitario, garantizar la legalidad y la intangibilidad del mismo, ejercer el control jurisdiccional sobre los actos que realizan los órganos que conforman el Sistema Andino de Integración, garantizar la observancia del ordenamiento jurídico andino y dirimir las controversias que se susciten derivadas de la aplicación de sus normas, de su validez, de su interpretación, de la omisión en el cumplimiento de las funciones de los órganos e instituciones comunitarios, y de las relaciones laborales entre estos últimos y sus empleados.

También el Tribunal es competente para dirimir mediante arbitraje, las controversias que se susciten por la aplicación o interpretación de contratos, convenios o acuerdos, suscritos entre órganos e instituciones del SAI o entre éstos y terceros, cuando las partes así lo acuerden. Asimismo, los particulares tendrán la opción de someter a arbitraje por el Tribunal, las controversias que ocurran por la aplicación o interpretación de aspectos contenidos en contratos de carácter privado y regidos por el ordenamiento jurídico de la Comunidad.

\footnotetext{
16 Kaune Arteaga, W. (2004). La necesidad de la integración y el orden y ordenamiento jurídico comunitario. En Tribunal de Justicia de la Comunidad Andina, op. cit.

17 Modificado por el Protocolo de Cochabamba, de mayo 28 de 1996.

18 Estatuto del Tribunal de Justicia de la Comunidad Andina. Artículo 4. "El Tribunal es el órgano jurisdiccional de la Comunidad Andina, de carácter supranacional y comunitario, instituido para declarar el derecho andino y asegurar su aplicación e interpretación uniforme en todos los Países Miembros. El Tribunal, en ejercicio de sus atribuciones, actuará salvaguardando los intereses comunitarios y los derechos que los Países Miembros poseen dentro del ordenamiento jurídico andino".
} 
De lo anterior se infiere la trascendencia del sistema de solución de controversias de la Comunidad Andina, el cual le da soporte institucional pues permite resolver, en distintas etapas y mediante diferentes mecanismos, las disputas que surgen entre los Países Miembros, entre éstos y los órganos de integración, incluyendo los casos en los cuales se encuentran vinculados intereses legítimos de los particulares. Por ello, la eficacia del sistema de solución de controversias andino constituye un instrumento de fortalecimiento del proceso de integración (Tangarife Torres, 2005, pp.331).

Funciones jurisdiccionales del Tribunal de Justicia de la Comunidad Andina: la interpretación prejudicial y la acción de incumplimiento. De las funciones jurisdiccionales del Tribunal de Justicia de la Comunidad Andina, se abordan: la interpretación prejudicial y la acción de incumplimiento (Secretaría General de la Comunidad Andina, 2008).

La interpretación prejudicial. Al igual que en el caso del Tribunal de Justicia de la Unión Europea, la interpretación prejudicial también llamada consulta prejudicial, figura propia del derecho comunitario, es la pieza clave del sistema jurisdiccional de la Comunidad Andina, por cuanto mediante dicho mecanismo el Tribunal asegura la aplicación uniforme de las normas que conforman el Ordenamiento Jurídico Andino y convierte automáticamente en jueces comunitarios a los jueces nacionales de los cuatro Países Miembros, estableciendo con ello una cooperación horizontal con los órganos jurisdiccionales nacionales.

De acuerdo con este procedimiento, los jueces o tribunales nacionales que conozcan un proceso en que deba aplicarse o se controvierta alguna de las normas del ordenamiento jurídico andino, deben solicitar la interpretación del Tribunal Andino de Justicia acerca del contenido y alcance de dichas normas, y en función de esa interpretación, apreciar los hechos y decidir la controversia (Secretaría General de la Comunidad Andina, 2008).

El juez o magistrado nacional podrá solicitar facultativamente, vía incidental, sin suspender el trámite procesal, siempre que esté conociendo de un proceso de primera instancia y que su sentencia sea susceptible de recursos en el derecho interno, una interpretación prejudicial; $y$ en el evento de tener que dictar sentencia sin que se hubiera recibido la interpretación del Tribunal Andino, deberá decidir ${ }^{19}$.

19 Tratado de Creación del Tribunal de Justicia de la Comunidad Andina, artículo 33, y Decisión 500 del Consejo de Ministros de Relaciones Exteriores, artículo 122. 
Por el contrario, la solicitud dejará de ser optativa y será obligatoria para el juez o magistrado nacional cuando conoce de un proceso de única o segunda instancia, y cuando su sentencia no sea susceptible de recurso alguno en el derecho interno. Esta solicitud de interpretación obligatoria que se genera de oficio o a petición de parte, corresponde también a un incidente, pero que en este caso suspende el trámite del proceso ${ }^{20}$.

La interpretación del Tribunal Andino, que deberá limitarse a precisar el contenido y alcance de las normas del ordenamiento comunitario referidas al caso concreto $^{21}$, se convierte, una vez recibida, en una obligación especial para el juez o magistrado consultante que deberá adoptar en su sentencia ${ }^{22}$.

Tremolada Álvarez (2006) explica que en todo caso, la oportunidad del juez o magistrado nacional para solicitar la interpretación prejudicial está dada, entre otras circunstancias, por el momento procesal interno del que dispone para tramitar el correspondiente incidente (p.70). $\mathrm{Al}$ respecto, el Tribunal de Justicia ha expresado que:

La solicitud de interpretación prejudicial y la petición de parte para que el juez nacional proceda a ella, se pueden hacer en cualquier estado y grado de la causa, pues lo que se plantea es una cuestión de mero derecho como es la interpretación de normas del ordenamiento jurídico del Acuerdo de Cartagena. Distinta es la solución en la legislación interna para las cuestiones de hecho en las cuales sí existe una oportunidad procesal para que las partes puedan promoverlas o presentarlas ante el juez nacional ${ }^{23}$.

De esta manera, como señala el Tribunal, se "consolida el principio de cooperación y colaboración entre el juez nacional y el juez comunitario en la administración de justicia, ya que ambos con jurisdicción y competencia propias efectúan su aporte a la vigencia del derecho de la integración" "24. En la misma línea, el Tribunal de Justicia de la Unión

20 Decisión 500 del Consejo de Ministros de Relaciones Exteriores, artículo 123.

21 El Tratado de Creación del Tribunal de Justicia, en el artículo 34 señala "en su interpretación el Tribunal deberá limitarse a precisar el contenido y alcance de las normas que conforman el ordenamiento de la Comunidad Andina, referida al caso concreto. El Tribunal no podrá interpretar el contenido y alcance del derecho nacional ni calificar los hechos materia del proceso, no obstante lo cual podrá referirse a éstos cuando ello sea indispensable a los efectos de la interpretación solicitada".

22 Tratado de Creación del Tribunal de Justicia de la Comunidad Andina, artículos 34 y 35 y Decisión 500 del Consejo de Ministros de Relaciones Exteriores, artículos 126 y 127.

23 Tribunal de Justicia de la Comunidad Andina. Sentencia 01-IP-87. G.O. No. 8 de 15 de febrero de 1988

24 Tribunal de Justicia de la Comunidad Andina. Sentencia 10-IP-94. G.O. No. 177 de 20 de abril de 1995. 
Europea ha dicho: "El órgano jurisdiccional nacional y el Tribunal de Justicia, en el orden de sus propias competencias, son llamados a contribuir directa y recíprocamente a la elaboración de una decisión" 25 .

La trascendencia de este mecanismo reposa en el hecho de que el ordenamiento jurídico comunitario es una normativa compleja, con características propias que lo distinguen de las normas nacionales y de las contenidas en los tratados internacionales de los que los Estados miembros son parte; y requiere por lo tanto que sea interpretado y aplicado de manera uniforme para asegurar así su supranacionalidad y su unidad; características esenciales del derecho comunitario junto con los principios de aplicación directa y con la primacía en relación con las normas nacionales.

Como destaca la Secretaría General de la Comunidad Andina (2008) con la interpretación prejudicial no se pretende unificar las legislaciones internas de los Países Miembros, sino que la norma sea interpretada de la misma manera y que aquellos tengan la misma percepción de lo quiso decir el legislador andino al crear la norma comunitaria (p.30).

La acción de incumplimiento. Es el mecanismo jurisdiccional que posibilita el control de cumplimiento de las normas que conforman el ordenamiento jurídico andino. Por medio de ella, se busca garantizar la observancia de los objetivos del proceso de integración, verificando el comportamiento de los Estados respecto de los compromisos asumidos desde la suscripción del Acuerdo de Cartagena ${ }^{26}$.

Esta acción consta de dos fases: una prejudicial y otra judicial (Secretaría General de la Comunidad Andina, 2008, p.21).

En la etapa prejudicial, el Estado o el particular que considere que otro Estado miembro ha incurrido en incumplimiento de obligaciones emanadas de las normas que conforman el ordenamiento jurídico de la Comunidad Andina, deberá agotar los trámites pre-contenciosos o administrativos en la Secretaría General, para que ésta realice las gestiones conducentes a subsanar el incumplimiento dentro de un plazo máximo de sesenta días, y una vez recibida la respuesta o vencido el plazo sin que se hubieren obtenido resultados positivos, la Secretaría

25 Sentencia de 1 de diciembre de 1965, Schwarze, 16/65, p. 1081.

26 Estatuto del Tribunal de Justicia de la Comunidad Andina, artículo 107: “Objeto y finalidad. La acción de incumplimiento podrá invocarse ante el Tribunal con el objeto de que un País Miembro, cuya conducta se considere contraria al ordenamiento jurídico comunitario, dé cumplimiento a las obligaciones y compromisos contraídos en su condición de miembro de la Comunidad Andina...". 
General de conformidad con su reglamento y dentro de los quince días siguientes, emitirá un dictamen motivado sobre el estado de cumplimiento de tales obligaciones.

La fase judicial se inicia en el caso de que el dictamen fuere de incumplimiento y el país requerido persistiere en la conducta objeto del reclamo, por lo que la Secretaría General deberá solicitar el pronunciamiento del Tribunal. Del mismo modo, si la Secretaría General no intentare la acción dentro de los sesenta días siguientes de emitido el dictamen de incumplimiento o no emitiere su dictamen dentro de los sesenta y cinco días siguientes a la fecha de presentación del reclamo, o el dictamen fuere de cumplimiento, el país o el particular reclamante podrá acudir directamente al Tribunal ${ }^{27}$.

El País Miembro cuya conducta haya sido declarada por el Tribunal como contraria al ordenamiento jurídico andino, quedará obligado a adoptar las medidas necesarias para su ejecución en un plazo no mayor de noventa días siguientes al de su notificación. Vencido este plazo sin que acate lo dispuesto en la sentencia, el Tribunal determinará los límites dentro de los cuales el país reclamante o cualquier otro País Miembro podrá restringir o suspender, total o parcialmente, las ventajas del Acuerdo de Cartagena que beneficien al País Miembro remiso. Para esto se tramitará el procedimiento sumario por incumplimiento de sentencia establecido en el Tratado de Creación del Tribunal y en su Estatuto ${ }^{28}$.

Respecto a la acción de incumplimiento, el Tribunal ha expresado que:

Sin perjuicio de la etapa prejudicial que se sustancia ante la Secretaría General, el control de la legitimidad de las actuaciones u omisiones de los Países Miembros frente al Derecho Comunitario corresponde en última instancia al Tribunal de Justicia de la Comunidad Andina, Órgano con la competencia exclusiva para declarar con autoridad de cosa juzgada judicial la existencia de un incumplimiento de las obligaciones impuestas a los Países Miembros en virtud del ordenamiento jurídico andino.... Aunque los motivos

27 Tratado de Creación del Tribunal de Justicia de la Comunidad Andina (artículos 23 y siguientes, Estatuto del Tribunal (artículos 107 y siguientes), y Decisión 425 del Consejo de Ministros de Relaciones Exteriores: Reglamento de Procedimientos Administrativos de la Secretaría General de la Comunidad Andina (artículos 56 y siguientes).

28 Tratado de Creación del Tribunal de Justicia Andino, art. 27 y Estatuto del Tribunal de Justicia de la Comunidad Andina, arts. 112 a 120. 
que contenga 'el dictamen, ${ }^{29}$ también deben mantener suficiente congruencia con los fundamentos de la demanda, pudiendo en consecuencia, ser enjuiciados por la parte demandada dentro del proceso de incumplimiento, la finalidad de esta acción, sin embargo, no se limita a un simple control de legalidad del dictamen, sino que se dirige a verificar si la infracción acusada fue o no cometida, y en definitiva a examinar las circunstancias del incumplimiento demandado y las causas exonerantes que pudiere haber propuesto la demandada como argumentos en su defensa ${ }^{30}$.

El ordenamiento jurídico andino admite la posibilidad de que el incumplimiento de un país miembro pueda ser demandado por personas naturales o jurídicas ejerciendo acciones judiciales ante sus jueces o tribunales nacionales competentes, de conformidad con su derecho interno, siempre que sus derechos resultaren afectados por la conducta en controversia. El juez nacional ejerce en estos eventos como juez comunitario. No obstante, con el fin de evitar conflictos de jurisdicción y competencia, está expresamente señalada ${ }^{31}$ la imposibilidad de ejercer simultáneamente acciones de incumplimiento ante el Tribunal Andino y los jueces o tribunales nacionales.

Asimismo y tal como lo pone de presente la Secretaría General de la Comunidad Andina (2008), con base en el Tratado de Creación del Tribunal $^{32}$, la sentencia de incumplimiento constituirá título legal y suficiente para que el particular pueda solicitar al juez nacional la indemnización de daños y perjuicios que correspondiere; así lo ha recogido el Tribunal en sentencias de los procesos 118-AI-03, 125-AI04 y $200-\mathrm{AI}-05^{33}$, entre otros, al recordar a los particulares acerca de la posibilidad de ejercer este derecho.

Como resultados parciales de la investigación, se presenta la población objeto de estudio, la cual comprende: las interpretaciones prejudiciales y acciones de incumplimiento resueltas en el período 2008-2012.

29 Emitido en la fase prejudicial por la Secretaría General de la Comunidad Andina.

30 Tribunal de Justicia de la Comunidad Andina. Sentencia emitida en el Proceso 43-AI-99, publicada en la G.O.A.C. No.620, de 23 de noviembre de 2000.

31 Tratado de Creación del Tribunal de Justicia de la Comunidad Andina, artículos 25 y 31.

32 Tratado de Creación del Tribunal de Justicia de la Comunidad Andina, art. 30.

33 Secretaría General de la Comunidad Andina. Op. cit., p. 23. 
Interpretaciones prejudiciales 2008 - 2010

\begin{tabular}{|c|c|c|c|}
\hline PAÍS & AÑO & NUMERO & TOTAL AÑO \\
\hline Bolivia & \multirow{4}{*}{2008} & 0 & \\
\hline Colombia & & 61 & \\
\hline Ecuador & & 22 & \\
\hline Perú & & 21 & 104 \\
\hline Bolivia & \multirow{4}{*}{2009} & 2 & \\
\hline Colombia & & 69 & \\
\hline Ecuador & & 26 & \\
\hline Perú & & 11 & 108 \\
\hline Bolivia & \multirow{4}{*}{2010} & 0 & \\
\hline Colombia & & 94 & \\
\hline Ecuador & & 7 & \\
\hline Perú & & 14 & 115 \\
\hline Bolivia & \multirow{4}{*}{2011} & 2 & \\
\hline Colombia & & 95 & \\
\hline Ecuador & & 32 & \\
\hline Perú & & 13 & 142 \\
\hline Bolivia & \multirow{4}{*}{2012} & 0 & \\
\hline Colombia & & 143 & \\
\hline Ecuador & & 12 & \\
\hline Perú & & 25 & 180 \\
\hline \multicolumn{3}{|l|}{ TOTAL } & 649 \\
\hline
\end{tabular}

Acciones de incumplimiento 2008 - 2012

\begin{tabular}{|l|c|r|l|}
\hline \multicolumn{1}{|c|}{ PAÍ́ } & AÑO & NUMERO & \multicolumn{1}{|c|}{ TEMA } \\
\hline Ecuador & 2008 & 1 & PROGRAMA DE LIBERACIÓN \\
\hline Ecuador & 2009 & 1 & PROGRAMA DE LIBERACIÓN \\
\hline Perú & 2010 & 1 & MEDIO AMBIENTE Y DESARROLLO RURAL \\
\hline Colombia & & 1 & PROPIEDAD INTELECTUAL \\
\cline { 1 - 1 } Colombia & \multirow{2}{*}{2011} & 1 & INTERPRETACION PREJUDICIAL \\
\cline { 1 - 1 } \cline { 3 - 4 } Perú & & 1 & MEDIO AMBIENTE DESARROLLO RURAL \\
\cline { 1 - 3 } Perú & 2012 & 1 & MEDIO AMBIENTE DESARROLLO RURAL \\
\hline
\end{tabular}

Como se puede observar, en el lapso comprendido en este trabajo, se resolvieron 649 interpretaciones prejudiciales y 7 acciones de incumplimiento.

En cuanto a las interpretaciones prejudiciales destaca que en el quinquenio, Colombia fue el País Miembro al cual el Tribunal de Justicia de la Comunidad Andina, resolvió más IP, un total de 462, seguido de Ecuador, con 99, Perú con 84 y en el extremo opuesto se sitúa Bolivia, con 4. 
Respecto de los temas abarcados en las IP destaca en primer lugar la propiedad intelectual ${ }^{34}, 587^{35}$; seguida de la interpretación prejudicial, 22 y del programa de liberación comercial, 20. En menor proporción, se identifican: inversiones, 5; liberalización del servicio de transporte, 3; comercio de bienes, 2; derecho comunitario andino, 2; aduanas, 2; política arancelaria,1; migración,1; sociolaboral, 1; sistema andino de calidad,1; convenios de complementación industrial, 1 y competencia del TJCA en materia laboral, 1.

Con referencia a las acciones de incumplimiento, en el período delimitado se resolvieron 7 en relación con medioambiente y desarrollo rural, programa de liberación, propiedad intelectual e interpretación prejudicial.

\section{CONCLUSIONES}

La Comunidad Andina, como proceso de integración, se fundamenta en el sentido de unidad entre los pueblos de los Países Miembros; en la aceptación de valores comunes; en el sentido de justicia social y equidad; y en la apertura al mundo y sus nuevas realidades.

El vínculo de pertenencia a esta Organización constituye un conjunto de relaciones y situaciones reguladas por un orden jurídico común, el derecho comunitario andino, el cual presenta unas características que le otorgan identidad propia, que por su efecto directo, pasa a ser compartido por los Países Miembros y por sus nacionales. De esta manera, tanto los países como sus ciudadanos, los particulares o las personas jurídicas se transforman en sujetos del nuevo sistema, en destinatarios de obligaciones y al mismo tiempo en titulares de derechos, como consecuencia del efecto de aplicación directa de la norma comunitaria.

La garantía de la interpretación y aplicación uniformes de este orden común, exige un órgano jurisdiccional también común, el Tribunal de Justicia de la Comunidad Andina. Esta instancia produce una jurisprudencia, la cual señala sus criterios interpretativos obligatorios.

34 Dentro de la propiedad intelectual, destaca las consultas resueltas en materia de propiedad industrial en torno a marcas, y patentes. Asimismo, se resolvieron IP acerca de diseño industrial, lema comercial, modelo de utilidad y competencia desleal.

35 En el período estudiado, se percibe un aumento en el número de IP resueltas con referencia a la propiedad intelectual, alcanzando a 160 en el año 2012. 
La jurisprudencia que se produce en el ordenamiento jurídico comunitario andino tiene dos clases de efectos y alcances: uno de carácter eminentemente judicial, generado dentro del proceso de aplicación directa de la norma comunitaria, cuando actúa y resuelve casos concretos sometidos a su decisión en el ejercicio de las acciones judiciales que le son propias, como la acción de incumplimiento; el otro, de carácter prejudicial e indirecto (en cuanto el Tribunal no aplica la norma comunitaria al caso concreto), que es el que se produce en la absolución de consultas prejudiciales, cuando le indica al juez nacional parámetros para interpretar la norma comunitaria que debe aplicar en la resolución de controversias sometidas a su jurisdicción.

De lo expuesto se desprende que el reto del jurista es conocer el ordenamiento jurídico andino, acatarlo y difundirlo para impulsar el sentido de pertenencia del ciudadano comunitario.

\section{REFERENCIAS BIBLIOGRÁFICAS}

ALADI. Recuperado de http://www.aladi.org/

Ander-Egg, E. (2004). Técnicas para la recogida de datos e información. En Métodos y técnicas de investigación social. Buenos Aires, Argentina: Grupo Editorial Lumen.

Antolínez Camargo, R., y Santamaría Velasco, F. (Comps.). (2011). La integración de América Latina y El Caribe: filosofía, geopolítica y cultura. Bogotá, D.C.: Ediciones USTA.

Ayala Mora, E. (2006). Enseñanza de Integración en los países andinos. Recuperado de http://www.comunidadandina.org/public/libro_53.htm

Base de datos documental del SELA. SELA. [Base de datos]. Caracas: Recuperado de http://www.sela.org/view/index.asp?ms=258\& pageMs $=4863$

Bernal, M., y Garnica, L.(2001). El Tribunal Andino de Justicia (trabajo de grado). Universidad Pontificia Javeriana, Bogotá, Colombia. Recuperado de http://www.javeriana.edu.co/biblos/tesis/derecho/ dere2/Tesis17.pdf

Blanco, C. (2011). Ordenamiento jurídico territorial colombiano Vs Ordenamiento jurídico andino. Revista Via Inveniendi et Iudicandi. (12), 
pp. 193-217. Recuperado de http://doctrina.vlex.com.co/vid/andinojuridical-ordering-andean-314322462

Cardona, D., Fuentes, A., Jaramillo, L. F., Ramírez Ocampo, J., Ramírez de Rincón, M., Reina, M., y Vacchino, J. M. (1992). Colombia y la Integración Americana. Bogotá, Colombia: CLADEI-FESCOL.

Centro de documentación INTAL (2009). Banco Interamericano de Desarrollo. [Base de datos]. Washington, D.C.: Recuperado de http://www.iadb.org/intal/intalcdi/ObrasDigitalizadas. aspx?lang=es

Centro de Estudios Latinoamericanos. Recuperado de http://catic.unab. edu.co/cel/

Comisión Económica para América Latina y el Caribe. Recuperado de http://www.cepal.org/

@CAN.Espacio virtual de trabajo de la Comunidad Andina. Recuperado de http://extranet.comunidadandina.org/ecan/

Comunidad Andina. Revista de la Integración. Recuperado de http://www.comunidadandina.org/Publicaciones.aspx?tipo=RE

Comunidad Andina. Recuperado de http://www.comunidadandina.org/

Constitución Política de Colombia. Bogotá, Colombia: Legis Editores S.A.

Cossette, P. (Trad. T. Bruckner). (2011). Diez reglas de la publicación en una revista académica. ¿Cómo llegar a ser un investigador convincente? (1 ed.). Bogotá, D.C.: Universidad de los Andes, Facultad de Administración. (Original en francés, 2011).

Díaz Müller, L. (1988). El derecho económico y la integración de América Latina. Bogotá, Colombia: TEMIS.

Dueñas Muñoz, J. (2011). ¿La interpretación prejudicial, piedra angular de la integración andina? Anuario de Derecho Constitucional Latinoamericano Año XVII, pp. 29-58. Recuperado de http:// www. juridicas.unam.mx/publica/librev/rev/dconstla/cont/2011/pr/pr4.pdf

Escribano Úbeda-Portugués, J. (2007). La consolidación del modelo europeo en el marco del nuevo regionalismo internacional. Madrid, 
España: Universidad Complutense de Madrid. Servicio de Publicaciones.

Escribano Úbeda-Portugués, J. (2011). Breve Introducción al Derecho Internacional Público. Madrid, España: Aebius.

EUR-LEX. El acceso al Derecho de la Unión Europea. Recuperado de http://eur-lex.europa.eu/es/tools/about.htm

Giacalone, R. (2002). La integración regional en la historia. Recuperado de http://www.comunidadandina.org/bda/docs/VE-INT0003.pdf

Giraldo Ángel, J., Giraldo López, M., y Giraldo López, A. (1999). Metodología y Técnica de la Investigación Sociojurídica (1 ed.). Colombia: Legis.

González, M. La interpretación prejudicial del Tribunal de Justicia de la CAN, como medio para la aplicación uniforme de la normativa comunitaria en materia de causales de irregistrabilidad de marcas (trabajo de grado, Especialización en Propiedad Intelectual). Universidad de los Andes, Bogotá, Colombia. Recuperado de http://tesis.ula.ve/postgrado/tde_busca/arquivo.php?codArquivo=6292

Hinojosa Martínez, L. M., y Roldán Barbero, J. (Coords.). Derecho Internacional Económico. Madrid, España: Marcial Pons Ediciones Jurídicas y Sociales S.A.

Hurtado de Barrera, J. (2002). El proyecto de investigación holística (1 ed.). Bogotá, Colombia: Cooperativa Editorial Magisterio.

Instituto de Relaciones Europeo-Latinoamericanas. (Julio 1999). Tres décadas de integración andina: logros y nuevos retos. Dossier (69).

Mangas Martín, A., y Liñán Nogueras, D. (2010). Instituciones y Derecho de la Unión Europea (6 ed.). Madrid, España: TECNOS.

Multilegis (2011). [Base de datos]. Bogotá, D.C.: LEGIS. Recuperado de http://www.multilegis.com.bases.unab.edu.co/colombia/unab/

Negro, S. (Dir.). (2010). Derecho de la Integración. MontevideoBuenos Aires: B def. 
Pico Mantilla, G. (2009). Jurisprudencia Andina. Recuperado de http://www.comunidadandina. org/bda/docs/CAN-INT-0035.pdf

Pico Mantilla, G. (2009). Temas jurídicos de la Comunidad Andina. Recuperado de http://www.comunidadandina.org/bda/docs/CAN-INT0038.pdf

Plata López, L., y Yepes Ceballos, D. (2009). Naturaleza jurídica de las normas comunitarias andinas. REVISTA DE DERECHO (31), pp. 96197. Recuperado de http://ciruelo.uninorte.edu.co/pdf/derecho/ 31/Revista\%20Derecho\%20No_31.pdf

Real Academia Española. Recuperado de http://www.rae.es/rae.html

Red Universitaria de Derecho Comunitario Andino. RUDCA. Recuperado de http://www.comunidadandina.org/rudca/index.html

Sáchica, L. C. (1990). Introducción al Derecho Comunitario Andino (2 ed.). Bogotá, Colombia: TEMIS.

Sarmiento, M. (2012). Efectos reglamentarios y jurisdiccionales del derecho comunitario andino sobre el derecho administrativo colombiano (tesis de Maestría). Universidad del Rosario, Bogotá, Colombia. Recuperado de http:// biblioteca.universia.net/ html_bura/ ficha/params/title/efectos-reglamentarios-jurisdiccionales-derechocomunitario-andino-derecho-administrativo-colombiano/id/ $55814730 . \mathrm{html}$

Secretaría General de la Comunidad Andina. (2006). La Comunidad Andina: una apuesta por nuestro futuro. Recuperado de http://www.comunidadandina.org/public/libro_CAN_nov 2006.pdf

Secretaría General de la Comunidad Andina. (2008). Manual de Procedimientos del Sistema Andino de Solución de Controversias (2 ed.). Lima, Perú: Secretaría General de la Comunidad Andina.

Secretaría General de la Comunidad Andina (Ed.). 40 Años de la Integración Andina Avances y Perspectivas. Revista de la Integración. Recuperado de http://www.comunidadandina.org/public/libro_ 100.htm 
Sistema de Información sobre Comercio Exterior. SICE. Recuperado de http://www. sice.oas.org/default_s.asp

Sistema Económico Latinoamericano y del Caribe. Boletín sobre integración latinoamericana y el Caribe. Recuperado de http://www. sela.org/view/index.asp? ms=258\&pageMs $=26260 \&$ alex_title=boletin de integracion\&alex_type $=\mathrm{S}$

Tangarife Torres, M. (2005). Derecho de la Integración en la Comunidad Andina (2 ed.). Bogotá D.C.: Cámara de Comercio de Bogotá.

Tremolada Álvarez, E. (2006). El derecho andino en Colombia (1 ed.). Bogotá, Colombia: Universidad Externado de Colombia.

Tribunal de Justicia de la Comunidad Andina. (Ed.). (2004). Testimonio Comunitario. Recuperado de http://www.tribunalandino.org.ec/ sitetjca/index.php

Tribunal de Justicia de la Comunidad Andina. Recuperado de http://www.tribunal andino.org.ec/sitetjca/index.php

Troconis Villarreal, M. (2004). Integración y Jurisdicción en la Comunidad Andina. En Tribunal de Justicia de la Comunidad Andina (ed.). Testimonio Comunitario. Recuperado de http://www.tribunal andino.org.ec/sitetjca/index.php

UNAB. Proyecto TEMA. Derecho de la Integración. Recuperado de http://tema.unabvirtual. edu.co/course/view.php?id=117

Unión Europea. Recuperado de http://europa.eu/index_es.htm

Uribe Restrepo, F. (1990). El derecho de la integración en el Grupo Andino. Quito, Ecuador: Tribunal de Justicia del Acuerdo de Cartagena.

vLex Colombia (2013). [Base de datos]. Colombia: Recuperado de http://vlex.com.bases.unab. edu.co/app

Von Bogdandy A., Landa Arroyo, C., y Morales Antoniazzi, M. (Eds.). (2009). Integración suramericana a través del Derecho? Un análisis interdisciplinario y multifocal. Madrid, España: Max-Planck-Institut Für Ausländisches Öffentliches Recht und Völkerreicht y Centro de Estudios Políticos y Constitucionales. 